\title{
Toll-like receptor 2 and -4 are involved in the pathogenesis of the Guillain-Barré syndrome
}

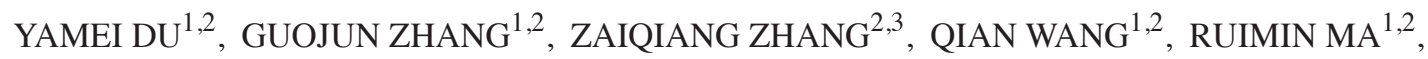 \\ LIMIN ZHANG ${ }^{1,2}$, FEI FAN ${ }^{1,2}$, YOURAN LI ${ }^{1,2}$, MENG WANG $^{1,2}$, HONG LV $^{1,2}$ and XIXIONG KANG ${ }^{1,2}$ \\ ${ }^{1}$ Laboratory Diagnosis Center; ${ }^{2}$ China National Clinical Research Center for Neurological Diseases; \\ ${ }^{3}$ Department of Neurology, Beijing Tiantan Hospital, Capital Medical University, Beijing 100050, P.R. China
}

Received July 29, 2014; Accepted April 15, 2015

DOI: $10.3892 / \mathrm{mmr} .2015 .3730$

\begin{abstract}
Guillain-Barré syndrome (GBS) is an autoimmune disorder of the peripheral nervous system characterized by weakness in the limbs. To date, numerous hypotheses have been suggested to explain the pathogenesis of GBS; however, the pathogenesis of GBS remains to be elucidated. The aim of the present study was to investigate the association between Toll-like receptor (TLR) 2, TLR4 and GBS. Therefore, the mRNA of TLR2, TLR4, myeloid differentiation factor(MyD)88 and nuclear factor (NF)- $\mathrm{BB}$ of peripheral blood mononuclear cells (PBMCs) in patients with GBS and healthy controls was assessed. To confirm the function of TLR2 and TLR4 in the pathogenesis of GBS, PBMCs derived from patients with GBS and healthy controls were cultured with various TLR agonists. The levels of tumor necrosis factor (TNF)- $\alpha$ and interleukin (IL)-1 $\beta$ were measured in the culture supernatant and fasting serum was obtained for the detection of anti-ganglioside antibodies. The results revealed that the mRNA levels of TLR2, TLR4, MyD88 and NF- $\mathrm{B}$ were significantly increased in patients with GBS compared with those in healthy controls $(\mathrm{P}=0.003,0.017,0.032$ and 0.015 , respectively). PBMCs from patients with GBS secreted higher levels of TNF- $\alpha$ and IL-1 $\beta$ than those from control subjects. The positive rate of immunoglobulin (Ig)G and IgM anti-ganglioside antibodies in patients with severe GBS was $42.86 \%$, which was markedly higher than rates found in patients with mild GBS (9.09 and 18.18\%, respectively). The results of the present study demonstrated that TLR2 and TLR4 are involved in the pathogenesis of GBS and that they and their associated signaling pathways may be targets for the treatment of GBS.
\end{abstract}

Correspondence to: Professor Guojun Zhang, Laboratory Diagnosis Center, Beijing Tiantan Hospital, Capital Medical University, 6 Tiantan Xili, Beijing 100050, P.R. China

E-mail: qdduckdymone@126.com

Key words: Guillain-Barré syndrome, Toll-like receptors, cytokines, anti-ganglioside antibody

\section{Introduction}

Guillain-Barré syndrome (GBS) is an autoimmune disorder, characterized by demyelination and infiltration of the peripheral nervous system by inflammatory cells, causing progressive weakness of the extremities. GBS consists of acute inflammatory demyelinating polyneuropathy, acute motor axonal neuropathy, acute motor sensory axonal neuropathy and Miller Fisher syndrome (MFS). The incidence of GBS has been reported to be $0.75-2$ cases per 100,000 individuals per year (1). In recent years, studies have reported that vaccines, such as the influenza A (H1N1) 2009 monovalent inactivated vaccine, are associated with a mildly increased risk of GBS $(2,3)$. However, the pathogenesis of GBS remains to be elucidated. To date, numerous hypotheses have been suggested to explain the pathogenesis of GBS, including the involvement of molecular mimicry, cytokines, anti-ganglioside antibodies and Toll-like receptors (TLRs). The present study was performed to investigate the association between TLRs and GBS.

TLRs are pattern recognition receptors, which recognize conserved motifs on pathogens via pathogen-associated molecular patterns (4). To date, 11 members of the TLR family have been identified in humans. Ligands of TLRs consist of endogenous and exogenous ligands. Each sub-type is able to recognize a specific ligand. For example, TLR2 recognizes peptidoglycan (PGN) (5) and TLR4 recognizes lipopolysaccharide (LPS) (6). After recognizing specific ligands, TLRs signal through myeloid differentiation factor (MyD)88-dependent or -independent pathways. TLR2 signals predominantly through a MyD88-dependent pathway, whereas TLR4 signals through the two types of pathway. Nuclear factor (NF)- $\kappa \mathrm{B}$ is a downstream signaling molecule of the MyD88-dependent pathway. Following signal transduction through the MyD88-dependent pathway, increased levels of $\mathrm{NF}-\kappa \mathrm{B}$ are generated. MyD88 and $\mathrm{NF}-\kappa \mathrm{B}$ are important in TLR signal transduction.

The major function of TLRs is to promoe the production of cytokines (7), including interleukin (IL)-1 $\beta$, IL-6, IL-12, tumor necrosis factor (TNF)- $\alpha$ and interferon (IFN)- $\gamma$. Cytokines are thought to be important in the pathogenesis of GBS (8), which suggests that TLRs may be involved in the pathogenesis of GBS. TLRs are also able to promote the differentiation and maturation of immune cells, which may also be associated with the pathogenesis of GBS. TNF- $\alpha$ and IL-1 $\beta$ are major 
pro-inflammatory cytokines secreted by T-helper (Th)1 cells. Numerous studies have demonstrated that the levels of TNF- $\alpha$ and IL-1 $\beta$ are significantly increased in the plasma and cerebrospinal fluid (CSF) of patients with GBS $(9,10)$. The current hypothesis states that TLRs are involved in the initiation of GBS. Following TLR activation, they increase NF- $\kappa \mathrm{B}$ levels through MyD88-dependent or -independent pathways, and finally induce the secretion of pro-inflammatory cytokines. To investigate the association between TLR 2 and TLR4 and GBS pathogenesis, TNF- $\alpha$ and IL-1 $\beta$ levels were measured in the culture supernatant of peripheral blood mononuclear cells (PBMCs) from patients with GBS and healthy controls in the present study.

Gangliosides have important biological functions, including cellular growth and differentiation, modulation of signal transduction and immune responses. GBS arises as a result of an autoimmune attack due to structural similarities between certain LPS molecules from Campylobacter jejuni and human nerve tissue gangliosides $(11,12)$. LPS is recognized by TLRs $(13)$ and therefore, TLRs may be involved in the pathogenesis of GBS. Recent evidence has suggested that anti-ganglioside antibodies are associated with the pathogenesis of GBS (14).

Excessive activation of TLRs is capable of eliminating auto-immune tolerance and may lead to autoimmune diseases (15), including systemic lupus erythematosus (SLE) and autoimmune hepatitis. Numerous studies have demonstrated that TLRs are involved in the pathogenesis of experimental autoimmune neuritis (EAN), an animal model of GBS $(16,17)$, and may be important in the pathogenesis of GBS.

To demonstrate the involvement of TLR2 and TLR4, as well as their signal transduction pathways, in the progression and pathogenesis of GBS, the mRNA, cytokines and anti-ganglioside antibodies of patients with GBS were analyzed.

\section{Patients and methods}

Patients and healthy controls. A total of 18 patients with GBS (11 patients with mild GBS and 7 patients with severe GBS) and 20 healthy controls were enrolled in the present study from the Health Examination Center of Beijing Tiantan Hospital (Beijing, China) between October 2012 and December 2013. All patients met the diagnostic criteria of GBS, and were classified into mild (1-3 points) and severe (4-6 points, including dysphagia and dyspnea) groups according to their Hughes scores (18). The exclusion criteria included patients with a fever or infection. The study was approved by the Ethics Committee of Beijing Tiantan Hospital, Capital Medical University (Beijing, China), and written informed consent was obtained from all participants.

Preparation of PBMCs. Approximately $15 \mathrm{ml} \mathrm{[3} \mathrm{ml}$ for reverse transcription-quantitative polymerase chain reaction (RT-qPCR) and $12 \mathrm{ml}$ for cell culture] of venous blood was collected from each subject into EDTA-anticoagulated vacuum tubes and PBMCs were isolated by Ficoll gradient separation with lymphocyte isolation agent (Sigma-Aldrich, St. Louis, MO, USA). Centrifugation was conducted for $30 \mathrm{~min}$ at $400 \mathrm{x} \mathrm{g}$.

RNA extraction. PBMCs were immediately snap-frozen in TRIzol reagent (Invitrogen Life Technologies, Carlsbad, CA, USA) following washing three times in phosphate-buffered saline (PBS; Invitrogen Life Technologies). Total RNA from PBMCs was extracted using TRIzol, absolute ethanol and isopropanol. The RNA concentration and quality were measured using the NanoDrop-1000 spectrophotometer (NanoDrop Technologies, Thermo Fisher Scientific, Waltham, MA, USA). An optical density of 1.8-2.1 at 260nm/280nm indicated high-quality RNA.

$R T$. A total of $20 \mu \mathrm{l}$ cDNA was prepared using a First Strand cDNA synthesis kit (Fermentas, Vilnius, Lithuania) from $1 \mu \mathrm{g}$ RNA. RT was performed according to the manufacturer's instructions. To ensure the success of RT, a general PCR was performed prior to RT-qPCR, which was performed under the same conditions. The general PCR was performed using 2X EasyTaq PCR Supermix (TransGen Biotech Co., Ltd., Beijing, China) using an ABI 9600 thermocycler (Applied Biosystems, Thermo Fisher Scientific). The amplification and detection stages of general PCR were performed as follows: $94^{\circ} \mathrm{C}$ for $5 \mathrm{~min}$ followed by 30 cycles of $94^{\circ} \mathrm{C}$ for $30 \mathrm{sec}, 58^{\circ} \mathrm{C}$ for $30 \mathrm{sec}$ and $72^{\circ} \mathrm{C}$ for $30 \mathrm{sec}$, followed by $72^{\circ} \mathrm{C}$ for $5 \mathrm{~min}$. The $20-\mu \mathrm{l}$ reaction mix consisted of RNase-free water $(7.8 \mu \mathrm{l})$, $0.6 \mu 110 \mu \mathrm{mol} / 1 \mathrm{GAPDH}_{1}$ forward/reverse primer, $1 \mu \mathrm{l} \mathrm{cDNA}$ and $10 \mu \mathrm{l}$ PCR mix. All primers were synthesized by Sangon Biotech (Shanghai, China) and are listed in Table I. The products were analyzed using agarose gel electrophoresis. The agarose gels were purchased from TransGen Biotech Co., Ltd., the electrophoresis meter used was the EPS 300 from Tanon Science \& Technology Co., Ltd. (Shanghai, China) and the BioSpectrum Imaging System was from UVP, LLC (Upland, CA, USA).

$q P C R$. qPCR was performed using the ABI Step-One Plus Real-Time PCR system (Applied Biosystems) using Power SYBR Green PCR Master mix (Applied Biosystems) according to the manufacturer's instructions. The sequences of the primers are shown in Table I. The amplification and detection stages of qPCR were performed as follows: $95^{\circ} \mathrm{C}$ for $10 \mathrm{~min}$ followed by 40 cycles of $95^{\circ} \mathrm{C}$ for $15 \mathrm{sec}, 58^{\circ} \mathrm{C}$ for $30 \mathrm{sec}$ and $72^{\circ} \mathrm{C}$ for $30 \mathrm{sec}$. The $20-\mu 1$ reaction system consisted of SYBR $\operatorname{mix}(10 \mu \mathrm{l}), 0.4 \mu \mathrm{l} 10 \mu \mathrm{mol} / 1$ forward/reverse primer, $1 \mu \mathrm{l}$ cDNA and 8.2 $\mu \mathrm{l}$ RNase-free water. Relative quantification of RNA expression was calculated using the $2^{-\Delta \mathrm{CT}}$ method using the following equation: $\Delta \mathrm{CT}=\mathrm{CT}_{\text {target gene }}-\mathrm{CT}_{\mathrm{GAPDH} 2}$. GAPDH was used as an internal control.

PBMC culture and cytokine analysis. PBMCs were isolated from $12 \mathrm{ml}$ venous blood from patients with GBS or healthy controls and were cultured with ligands of TLR2 (PGN; Sigma-Aldrich; $100 \mu \mathrm{g} / \mathrm{ml}$ ) or TLR4 (LPS; Sigma-Aldrich; $10 \mu \mathrm{g} / \mathrm{ml})$. PBMCs cultured without ligands were used as blank controls. Subsequently, $500 \mu \mathrm{l}$ PBS was added to dilute the PBMCs. The cell concentration was determined using the Sysmex XT-4000i hematology analyzer (Sysmex, Kobe, Japan). PBMCs were cultured in 24 well-plates and each well consisted of RPMI 1640 medium supplemented with $10 \%$ fetal calf serum (Invitrogen Life Technologies), $10^{6}$ PBMCs and the respective agonist. Following incubation for $24 \mathrm{~h}$ in an SS-23062 carbon dioxide incubator (SHEL LAB, Cornelius, OR, USA) with $5 \% \mathrm{CO}_{2}$, the supernatant was harvested, and TNF- $\alpha$ and IL- $1 \beta$ were measured using the IMMULITE 1000 immunoassay system (Siemens Healthcare Diagnostics, 


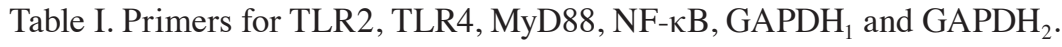

\begin{tabular}{|c|c|c|c|}
\hline Genes & Forward primers & Reverse primers & $\begin{array}{l}\text { Product } \\
\text { length (bp) }\end{array}$ \\
\hline TLR2 & 5'-GCCTCTCCAAGGAAGAATCC-3' & 5'-TCCTGTTGTTGGACAGGTCA-3' & 144 \\
\hline TLR4 & 5'-AAGCCGAAAGGTGATTGTTG-3' & 5'-CTGAGCAGGGTCTTCTCCAC-3' & 153 \\
\hline MyD88 & 5'-CATCACCACACTTGATGACCC-3' & 5'-TGCACAAACTGGATGTCGC-3' & 90 \\
\hline $\mathrm{NF}-\kappa \mathrm{B}$ & 5'-AGCCTGGTAGACACGTACCG-3' & 5'-CCGTACGCACTGTCTTCCTT-3' & 200 \\
\hline $\mathrm{GAPDH}_{1}$ & 5'-GTCACCAGGGCTGCTTTT-3' & 5'-CATCACGCCACAGTTTCC-3' & 544 \\
\hline $\mathrm{GAPDH}_{2}$ & 5'-TCGGAGTCAACGGATTTGG-3' & 5'-GCAACAATATCCACTTACCAGAGTTAA-3' & 79 \\
\hline
\end{tabular}

$\mathrm{GAPDH}_{1}$ was used for general PCR and $\mathrm{GAPDH}_{2}$ for reverse transcription quantitative PCR. PCR, polymerase chain reaction; TLR, Toll-like receptor; NF, nuclear factor; MyD, myeloid differentiation factor.

Erlangen, Germany) according to the manufacturer's instructions.

Anti-ganglioside antibody detection. Serum anti-ganglioside antibodies were assessed using an immunoblot assay in 18 patients with GBS and 20 healthy controls using EUROLINE anti-ganglioside profile 2: IgG and IgM (EUROIMMUN Medizinische Labordiagnostika AG, Lübeck, Germany).

Statistical analysis. All values are expressed as the mean \pm standard error. Comparisons of mRNA levels between patients with GBS and healthy controls were performed using Student's t-test. The Fisher exact probability test was used to examine the correlation between positive anti-ganglioside antibody rate and mild or severe GBS. SPSS software, version 17.0 (SPSS, Inc., Chicago, IL, USA) was used for statistical analysis. $\mathrm{P}<0.05$ was considered to indicate a statistically significant difference.

\section{Results}

Analysis of TLR2, TLR4, MyD88 and NF- $\kappa B$ mRNA. A previous study demonstrated that TLRs and their signal transduction pathway may be involved in the pathogenesis of GBS (19). In the present study, PCR assays were performed using PBMCs from patients with GBS and healthy controls to analyze the mRNA levels of TLR2, TLR4, MyD88 and $\mathrm{NF}-\kappa \mathrm{B}$. As shown in Fig. 1, TLR2, TLR4, MyD88 and NF- $\kappa \mathrm{B}$ mRNA levels were significantly upregulated in patients with GBS compared with those in healthy controls $(\mathrm{P}<0.05)$.

The correlation of mRNA expression and the severity of GBS was evaluated using Student's t-test. As shown in Fig. 2, no significant difference was identified in TLR2, TLR4 and $N F-\kappa B$ mRNA expression between the two groups, although the levels tended to be higher in patients with severe GBS $(P>0.05)$. In addition, no significant difference was identified in MyD88 levels between patients with mild or severe GBS.

Analysis of cytokines secreted by stimulated PBMCs. GBS is a T-helper (Th)1-type autoimmune disease. TNF- $\alpha$ and IL-1 $\beta$ are major pro-inflammatory cytokines released by Th1 cells. It was hypothesized that TLRs are involved in the initiation of GBS, and that following activation, TLRs are able to promote

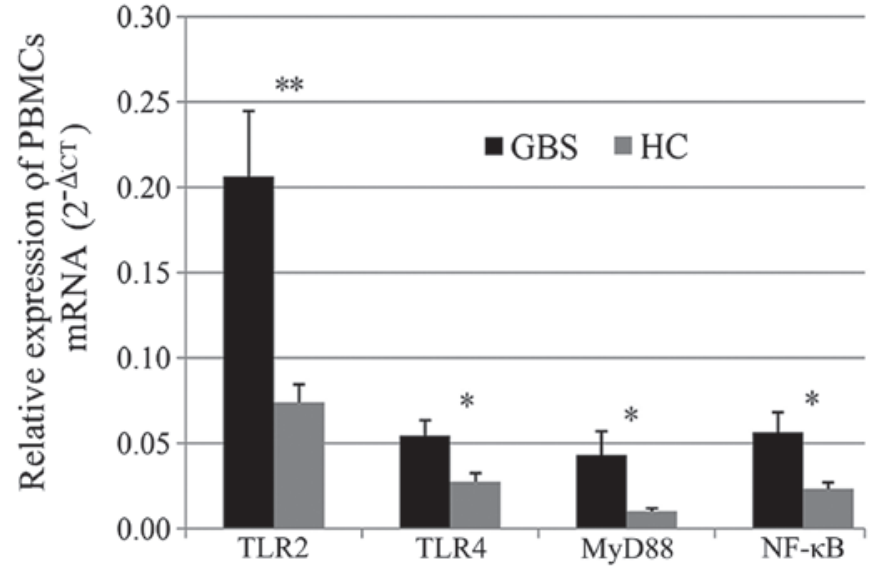

Figure 1. Relative expression of TLR2, TLR4, MyD88 and NF- $\kappa$ B mRNA. Values are expressed as the mean \pm standard error. Expression levels of TLR2, TLR4, MyD88 and NF- $\mathrm{B}$ mRNA increased significantly in patients with GBS compared with those in healthy controls $\left({ }^{*} \mathrm{P}<0.05 ;{ }^{* *} \mathrm{P}<0.01\right.$ for comparison between GBS and HC groups). $\mathrm{P}_{\mathrm{TLR} 2}=0.003, \mathrm{P}_{\mathrm{TLR} 4}=0.017$, $\mathrm{P}_{\mathrm{MyD} 88}=0.032$ and $\mathrm{P}_{\mathrm{NF}-\mathrm{kB}}=0.015$. GBS, Guillain-Barré syndrome; HC, healthy controls; TLR, Toll-like receptor; NF, nuclear factor; MyD, myeloid differentiation factor; PBMCs, peripheral blood mononuclear cells; CT, cycle threshold.

increased NF- $\kappa \mathrm{B}$ levels through MyD88-dependent or -independent pathways, to induce the secretion of pro-inflammatory cytokines, including TNF- $\alpha$ and IL-1 $\beta$. To further investigate the association between TLR2 and TLR4, and GBS pathogenesis, TNF- $\alpha$ and IL-1 $\beta$ levels were measured in PBMC culture supernatants. PBMCs from patients with GBS secreted higher levels of TNF- $\alpha$ when stimulated with LPS or PGN compared with those of healthy controls (Fig. 3A). IL-1 $\beta$ secretion by PBMCs from patients with GBS was significantly higher in the unstimulated or LPS-stimulated groups compared with that of healthy controls $(\mathrm{P}<0.05$ and $\mathrm{P}<0.01$, respectively) (Fig. 3B). However, no significant difference was identified between patients with GBS and healthy controls when PBMCs were stimulated with PGN (Fig. 3B). Compared with the blank control, the secretion of TNF- $\alpha$ and IL-1 $\beta$ was significantly increased in PBMCs from patients with GBS when stimulated with PGN ( $<<0.001)$ (Fig. 4A). Similarly, stimulation with LPS significantly increased the secretion of TNF- $\alpha$ and IL-1 $\beta$ by PBMCs compared with those in the control group $(\mathrm{P}<0.05$ 
A

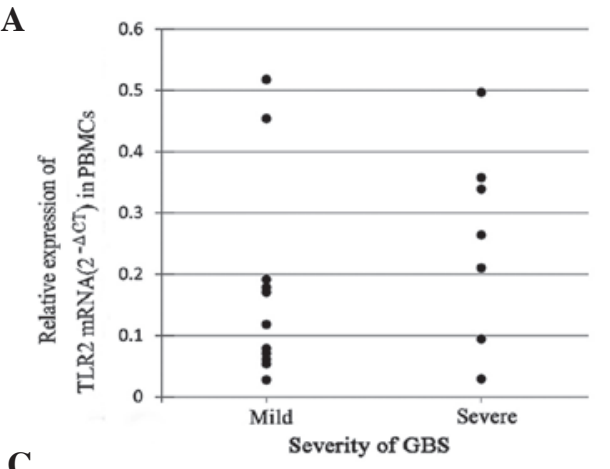

C

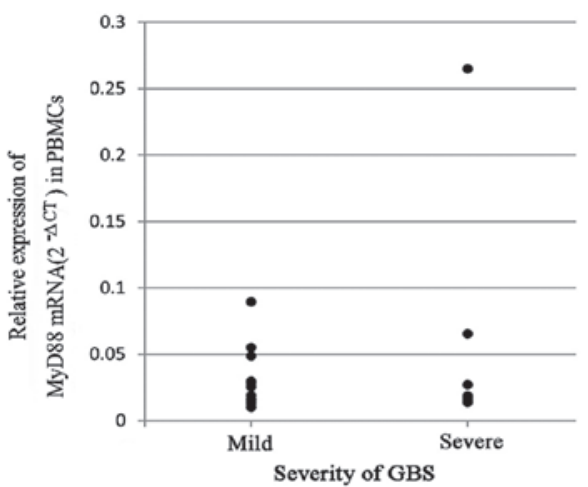

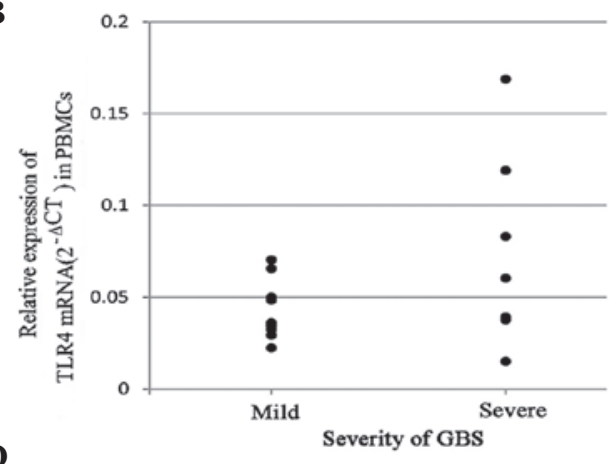

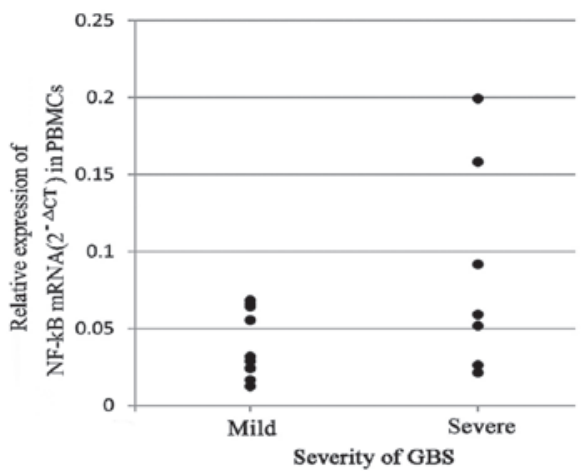

Figure 2. Expression levels of (A) TLR2 ( $\mathrm{P}=0.317)$, (B) TLR4 ( $\mathrm{P}=0.158)$, (C) MyD88 ( $\mathrm{P}=0.338$ ) and (D) NF-kB ( $\mathrm{P}=0.103)$ in PBMCs of patients with mild or severe Guillain-Barré syndrome. P-values refer to the comparison between mild and severe GBS. TLR, Toll-like receptor; NF, nuclear factor; MyD, myeloid differentiation factor; GBS, Guillain-Barré syndrome; PBMCs, peripheral blood mononuclear cells; CT, cycle threshold.
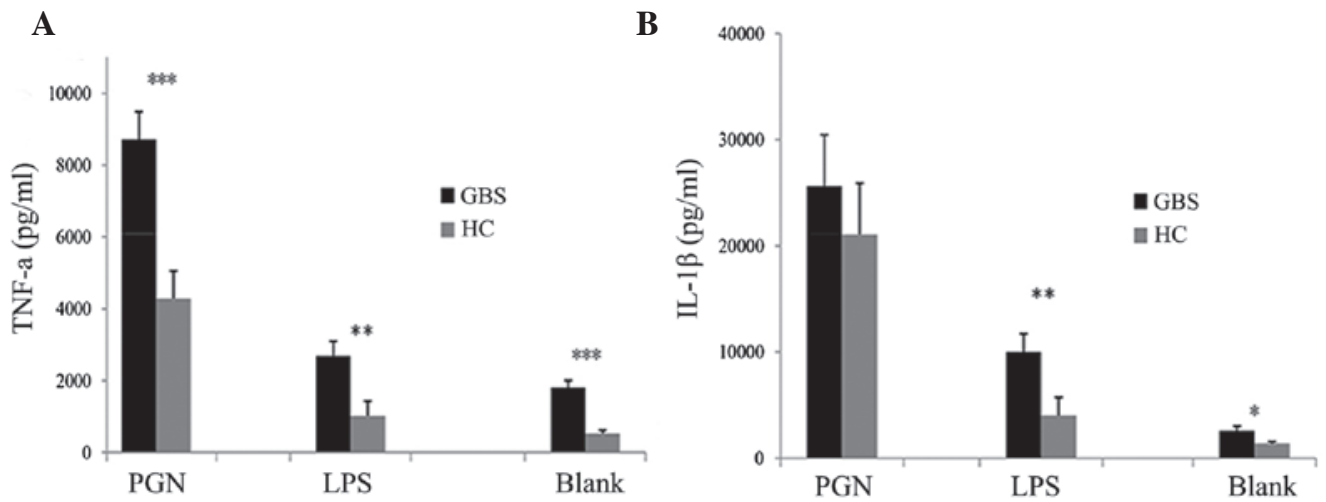

Figure 3. Secretion of (A) TNF- $\alpha$ and (B) IL-1 $\beta$ by peripheral blood mononuclear cells from patients with Guillain-Barré syndrome and healthy controls. Values are expressed as the mean \pm standard error. ${ }^{*} \mathrm{P}<0.05,{ }^{* *} \mathrm{P}<0.01,{ }^{* * *} \mathrm{P}<0.001$ for comparison between GBS and HC groups. LPS, lipopolysaccharide; PGN, peptidoglycan; TNF, tumor necrosis factor; IL, interleukin; GBS, Guillain-Barré syndrome; HC, healthy controls.

A

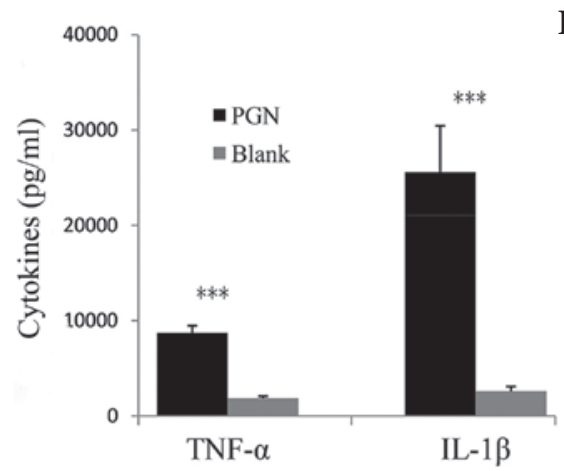

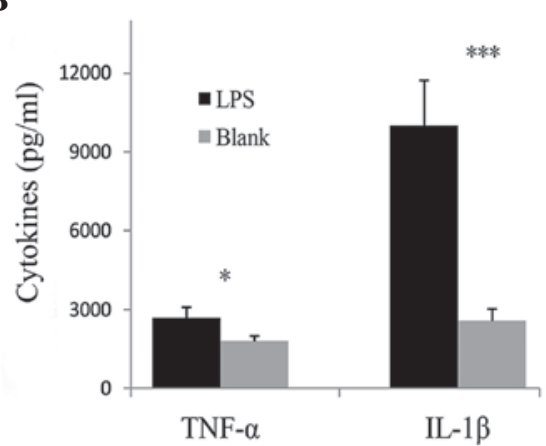

Figure 4. Secretion of TNF- $\alpha$ and IL-1 $\beta$ by peripheral blood mononuclear cells from patients with Guillain-Barré syndrome stimulated with (A) PGN or (B) LPS compared with blank controls. Values are expressed as the mean \pm standard error. ${ }^{*} \mathrm{P}<0.05,{ }^{* * *} \mathrm{P}<0.01,{ }^{* * * *} \mathrm{P}<0.001$ for comparison between stimulated and blank groups. LPS, lipopolysaccharide; PGN, peptidoglycan; TNF, tumor necrosis factor; IL, interleukin. 
Table III. Anti-ganglioside antibody-positive rate in patients with mild $(n=11)$ or severe $(n=7)$ Guillain-Barré syndrome.

\begin{tabular}{lcc}
\hline Severity & $\operatorname{IgG}(\%)$ & $\operatorname{IgM}(\%)$ \\
\hline Mild & 9.09 & 18.18 \\
Severe & 42.86 & 42.86
\end{tabular}

Ig, immunoglobulin.

and $\mathrm{P}<0.001$, respectively) (Fig. 4B). The results revealed an increased activation of TLR2 and TLR4 in patients with GBS. As shown in Fig. 3, the release of the cytokines TNF- $\alpha$ and IL-1 $\beta$ was increased in the PBMCs of patients with GBS under basal conditions (blank) compared with that in healthy controls, which suggests an enhanced sensitivity of the PBMCs in patients with GBS. The increased activation and enhanced sensitivity of the PBMCs may be somewhat important for the induction and/or progression of GBS.

Anti-ganglioside antibody measurement. To examine the association between TLRs and GBS indirectly, serum anti-ganglioside antibodies were measured using the EUROLINE anti-ganglioside profile 2 . The total positive rate of patients with GBS was significantly higher than that of healthy controls $(\mathrm{P}<0.05$; Table II), which is in line with a previous study (20). Among 11 patients with mild GBS, 1 patient was IgG-positive, while 2 other patients were IgM-positive. By contrast, there were 3 IgG-positive and $3 \mathrm{IgM}$-positive patients in the group of patients with severe GBS. In addition, 2 of the 3 IgG-positive GBS patients were additionally IgM-positive. As shown in Table III, IgG and IgM anti-ganglioside antibody-positive rates were higher in patients with severe GBS than those in patients with mild GBS, although no statistically significant difference was identified. These results were consistent with the results regarding the expression of TLR2, TLR4, MyD88 and NF- $\mathrm{kB}$, which indicated that TLRs and their signaling pathways may be involved in the pathogenesis of GBS.

\section{Discussion}

GBS presents as a weakness of the extremities and may progress to weakness of the trunk, cervical area, facial muscles and even respiratory muscles, causing respiratory failure in severe cases (21). Patients with GBS are often treated with hormones or plasma exchange. However, the effects of treatments remain poor. Thus, it is important to investigate the pathogenesis of GBS, in order to develop beneficial and efficacious treatments for patients with GBS. Previous studies indicated that TLRs, such as SLE, are significant in autoimmune diseases $(22,23)$, primary biliary cirrhosis and autoimmune hepatitis (22). Several studies also demonstrated that the expression of TLRs was increased in EAN rats and patients with GBS. The present study identified that TLR2 and TLR4 and their signaling pathways are functionally involved in the pathogenesis of GBS.

Gries et al (24) systematically examined the expression patterns of TLRs in sciatic nerves and lymphoid organs during 
the course of murine EAN, and observed a pronounced upregulation of TLR2, TLR6 and TLR11 on T cells and TLR4 and TLR6 on antigen-presenting cells. Another study reported that MyD88 (-/-) mice were completely EAN-resistant and TLR4 (-/-) and TLR9 (-/-) mice exhibited markedly more severe EAN symptoms than wild-type mice (17). This indicated that TLR4, TLR9 and MyD88 are important in the pathogenesis of EAN. In patients with GBS, Wang et al (19) found that TLR2, TLR4 and TLR9 mRNA levels were significantly increased. In accordance with the results of studies using the murine EAN model, it was suggested that TLR2, TLR4 and TLR9 may be involved in the pathogenesis of GBS.

In line with investigations into EAN and GBS, it was demonstrated that TLR2 and TLR4 mRNA expression was significantly elevated in PBMCs from patients with GBS. To further investigate the association between TLRs and GBS, the mRNA levels of MyD88 and NF- $\kappa B$ were measured using RT-qPCR. MyD88 and NF- $\mathrm{kB}$ are two key molecules of the TLR signaling pathways. As expected, marked increases in MyD88 and NF- $\kappa B$ mRNA levels were observed in patients with GBS compared with those in healthy controls. This indicated that TLR2, TLR4 and their signaling pathways are functionally involved in the pathogenesis of GBS.

Previous studies have described contradictory results regarding the association between TLRs and GBS severity. Wang et al (19) indicated that TLR mRNA levels had a marked positive correlation with the disability scores of patients with GBS. However, another study reported that the severity of clinical symptoms had a negative correlation with TLR2, TLR4 and TLR6 mRNA expression levels (24). Patients with GBS were divided into two groups (mild and severe) according to their clinical manifestation, and their mRNA levels were compared using Student's t-test. In accordance with the findings of Wang et al (19), the results of the current study demonstrated the same trend of alterations between TLR2, TLR4 and NF- $\kappa$ B mRNA and the condition of the patients with GBS. However, no significant difference was identified in MyD88 mRNA levels between patients with mild and severe GBS. This may be the result of an insufficient sample size. A larger cohort of patients with GBS is required to investigate this condition further.

It was hypothesized that GBS is a Th1-type autoimmune disease, and that Th1 cytokines, including IL- $1 \beta$, TNF- $\alpha$ and IFN- $\gamma$, are significantly correlated with GBS pathogenesis. Matsui et al (25) reported that IL-1 $\beta$, TNF- $\alpha$ and IFN- $\gamma$ were increased in the spinal cord of EAN rats, whereas anti-inflammatory cytokines, including IL-4 and IL-10, were decreased (25). Serum IFN- $\gamma$ was also elevated in patients with GBS (26). TNF- $\alpha$ is able to inhibit Schwann cell proliferation and mediate Schwann cell death (27). TNF- $\alpha$ was elevated and associated with disease severity in EAN (28) and GBS (29). In a further study, Zhang et al (30) observed that the onset of EAN in TNF- $\alpha$ gene-deficient mice was markedly later than that in wild type mice. In GBS, an increased expression of IL-1 $\beta$ was immunolocalized on Schwann cell membranes in sural nerve biopsies (31) and IL-1 $\beta$ was detected in the cerebrospinal fluid (32). These findings indicated that TNF- $\alpha$, IL- $1 \beta$ and IFN- $\gamma$ are involved in the pathogenesis of GBS. Therefore, in the present study, PBMCs from patients with GBS and healthy controls were cultured and TNF- $\alpha$ and
IL-1 $\beta$ levels were measured in the supernatants. The results indicated that, LPS-stimulated PBMCs from patients with GBS secreted more TNF- $\alpha$ and IL- $1 \beta$ than those of healthy controls. This indicated that excessive activation of TLRs was involved in the pathogenesis of GBS. When stimulated by PGN or LPS, PBMCs from patients with GBS secreted more TNF- $\alpha$ and IL- $1 \beta$ than blank controls. Therefore, the present study provided evidence supporting the hypothesis that TLR2 and TLR4 are functionally involved in the pathogenesis of GBS.

In the majority of previous studies, anti-ganglioside antibodies have been found in autoimmune neuropathies, particularly GBS $(33,34)$. It was hypothesized that different sub-types of anti-ganglioside antibodies are associated with different GBS subtypes (35). Anti-GM1 antibodies are commonly associated with a pure motor variant of GBS (36), and GQ1b may be a target antigen responsible for patients with MFS (37). A study reported that anti-ganglioside antibodies are able to inhibit axon regeneration through neuronal gangliosides independent of endogenous regeneration inhibitors (38). Gangliosides have biological functions, including cellular growth and differentiation, modulation of signal transduction and immune reactions. Sheikh and Zhang (39) found that anti-ganglioside antibodies induced damage to intact nerve fibers and inhibited axon regeneration via an $\mathrm{Fc} \gamma$ receptor. Based on these studies, it was hypothesized that anti-ganglioside antibodies may be associated with the severity of GBS. The present results revealed that positive anti-ganglioside antibody rates were significantly increased in patients with GBS. In addition, positive anti-ganglioside antibody rates were higher in patients with severe GBS compared with those with mild GBS, although no significant difference was identified. This revealed that anti-ganglioside antibodies may be associated with the severity of GBS; however, larger numbers of patients with GBS are required to verify these results in for future studies. The antibody-positive rates of the mild and severe patients with GBS are in accordance with the results regarding the mRNA levels of TLR2, TLR4, MyD88 and NF- $\mathrm{KB}$, which further suggests that TLR2, TLR4 and their signaling pathways are involved in the pathogenesis of GBS.

The results of the present study revealed that TLR2, TLR4, MyD88 and NF- $k B$ mRNA increased significantly in GBS, and that activated TLR2 and TLR4 are able to promote the abnormal secretion of TNF- $\alpha$ and IL- $1 \beta$. However, anti-ganglioside antibodies produced due to molecular mimicry were shown to be significantly associated with GBS and the severity of GBS. In conclusion, the present study further supported the hypothesis that TLR2, TLR4 and their signaling pathways are functionally involved in the pathogenesis of GBS. The present study suggested that TLR2, TLR4 and their signaling pathways may be potential treatment targets, which requires further investigation.

\section{Acknowledgements}

The present study was supported by Natural Science Foundation of Beijing (grant no. 7142051), the High Level Technical Talent Development of Beijing Health System (grant no. 2013-3-052) and the National Key Technology Research and Development Program of 
the Ministry of Science and Technology of China (grant no. 2013BAI09B03).

\section{References}

1. McGrogan A, Madle GC, Seaman HE and de Vries CS: The epidemiology of Guillain-Barŕe syndrome worldwide. A systematic literature review. Neuroepidemiology 32: 150-163, 2009.

2. Huang WT, Yang HW, Liao TL, et al: Safety of pandemic (H1N1) 2009 monovalent vaccines in taiwan: a self-controlled case series study. PLoS One 8: e58827, 2013.

3. Salmon DA, Proschan M, Forshee R, et al: Association between Guillain-Barré syndrome and influenza A (H1N1) 2009 monovalent inactivated vaccines in the USA: a meta-analysis. Lancet 381: 1461-1468, 2013.

4. Zhang D, Zhang G, Hayden MS, et al: A toll-like receptor that prevents infection by uropathogenic bacteria. Science 303: 1522-1526, 2004.

5. Eom SH, Gu GJ, Suh CW, et al: Suppression of inducible nitric oxide synthase expression induced by Toll-like receptor agonists by (E)-1-(2-(2-nitrovinyl)phenyl) pyrrolidine. Int Immunopharmacol 17: 205-209, 2013

6. Santaolalla R, Sussman DA, Ruiz JR, et al: TLR4 activates the $\beta$-catenin pathway to cause intestinal neoplasia. PLoS One 8 : e63298, 2013.

7. Arbour NC, Lorenz E, Schutte BC, et al: TLR4 mutations are associated with endotoxin hyporesponsiveness in humans. Nat Genet 25: 187-191, 2000.

8. Zhang ZY, Zhang Z and Schluesener HJ: FTY720 attenuates lesional interleukin-17 (+) cell accumulation in rat experimenta autoimmune neuritis. Neuropathol Appl Neurobiol 35: 487-495, 2009.

9. Zhang ZY, Zhang Z and Schluesener HJ: Toll-like receptor-2, CD14 and heat-shock protein 70 in inflammatory lesions of rat experimental autoimmune neuritis. Neuroscience 159: 136-142, 2009.

10. Reynolds JM, Pappu BP, Peng J, et al: Toll-like receptor 2 signaling in CD4(+) $\mathrm{T}$ lymphocytes promotes $\mathrm{T}$ helper 17 responses and regulates the pathogenesis of autoimmune disease. Immunity 32: 692-702, 2010.

11. Islam Z, Gilbert M, Mohammad QD, et al: Guillain-Barré syndrome-related Campylobacter jejuni in Bangladesh: ganglioside mimicry and cross-reactive antibodies. PLoS One 7: e43976, 2012.

12. Heikema AP, Koning RI, Duarte dos Santos Rico S, et al: Enhanced sialoadhesin-dependent uptake of Guillain-Barre syndrome-associated Campylobacter jejuni strains by human macrophages. Infect Immun 81: 2095-2103, 2013.

13. Nakamura M, Funami K, Komori A, et al: Increased expression of Toll-like receptor 3 in intrahepatic biliary epithelial cells at sites of ductular reaction in diseased livers. Hepatol Int 2: 222-230, 2008.

14. Kaida K, Ariga T and Yu RK: Antiganglioside antibodies and their pathophysiological effects on Guillain-Barr e syndrome and related disorders-A review. Glycobiology 19: 676-692, 2009.

15. Hans M and Hans VM: Toll-like receptors and their dual role in periodontitis: a review. J Oral Sci 53: 263-271, 2011.

16. Deng YN and Zhou WB: Expression of TLR4 and TLR9 mRNA in Lewis rats with experimental allergic neuritis. Neuroimmunomodulation 14: 337-343, 2007.

17. Marta M, Andersson A, Isaksson M, et al: Unexpected regulatory roles of TLR4 and TLR9 in experimental autoimmune encephalomyelitis. Eur J Immunol 38: 565-575, 2008.

18. van Koningsveld R, Steyerberg EW, Hughes RA, et al: A clinical prognostic scoring system for Guillain-Barré syndrome. Lancet Neurol 6: 589-594, 2007.

19. Wang YZ, Liang QH, Ramkalawan $\mathrm{H}$, et al: Expression of Toll-like receptors 2, 4 and 9 in patients with Guillain-Barré syndrome. Neuroimmunomodulation 19: 60-68, 2012.
20. Kusunoki S: Antiglycolipid antibodies in Guillain-Barré syndrome and autoimmune neuropathies. Am J Med Sci 319: 234-239, 2000

21. Arcila-Londono X and Lewis RA: Guillain-Barré syndrome. Semin Neurol 32: 179-186, 2012.

22. Komatsuda A, Wakui H, Iwamoto K, et al: Up-regulated expression of Toll-like receptors mRNAs in peripheral blood mononuclear cells from patients with systemic lupus erythematosus. Clin Exp Immunol 152: 482-487, 2008.

23. Papadimitraki ED, Choulaki C, Koutala E, et al: Expansion of toll-like receptor 9-expressing B cells in active systemic lupus erythematosus: implications for the induction and maintenance of the autoimmune process. Arthritis Rheum 54: 3601-3611, 2006.

24. Gries M, Davies L, Liu Y, Bachhuber A, et al: Response of Toll-like receptors in experimental Guillain-Barré syndrome: a kinetic analysis. Neurosci Lett 518: 154-160, 2012.

25. Matsui H, Ohgomori T, Natori T, et al: Keratan sulfate expression in microglia is diminished in the spinal cord in experimental autoimmune neuritis. Cell Death Dis 4: e946, 2013.

26. Orlikowski D, Chazaud B, Plonquet A, et al: Monocyte chemoattractant protein 1 and chemokine receptor CCR2 productions in Guillain-Barré syndrome and experimental autoimmune neuritis. J Neuroimmunol 134: 118-127, 2003.

27. Boyle K, Azari MF, Cheema SS and Petrato SS: TNF alpha mediates Schwann cell death by upregulating p75NTR expression without sustained activation of NFkappaB. Neurobiol Dis 20: 412-427, 2005.

28. Kurz M, Pischel H, Hartung HP and Kieseier BC: Tumor necrosis factor-alpha-converting enzyme is expressed in the inflamed peripheral nervous system. J Peripher Nerv Syst 10: 311-318, 2005.

29. Zhang J, Dong H, Li B, et al: Association of tumor necrosis factor polymorphisms with Guillain-Barré syndrome. Eur Neurol 58: 21-25, 2007.

30. Zhang HL, Hassan MY, Zheng XY, et al: Attenuated EAN in TNF- $\alpha$ deficient mice is associated with an altered balance of M1/M2 macrophages. PLoS One 7: e38157, 2012.

31. Hayashi R, Xiao W, Kawamoto M, et al: Systemic glucocorticoid therapy reduces pain and the number of endoneurial tumor necrosis factor-alpha (TNF alpha)-positive mast cells in rats with a painful peripheral neuropathy. J Pharmacol Sci 106: 559-565, 2008.

32. Sivieris, Ferrarini AM, Lolli F, et al: Cytokine pattern in the cerebrospinal fluid from patients with GBS and CIDP. J Neurol Sci 147: 93-95, 1997.

33. Vaishnavi C, Behura C, Prabhakar S, Sharma A and Kharbanda P: Anti-ganglioside antibodies in patients with Guillain Barré syndrome and other neurological disorders. Indian J Med Microbiol 31: 177-179, 2013.

34. Chatani H, Tanaka M, Nagata T, et al: Guillain-Barré syndrome-like-onset neurosarcoidosis positive for immunoglobulin G anti-N-acetylgalactosaminyl-GDla antibody. J Clin Neurosci 21: 170-172, 2014

35. Kaida K, Ariga T and Yu RK: Antiganglioside antibodies and their pathophysiological effects on Guillain-Barré syndrome and related disorders-a review. Glycobiology 19: 676-692, 2009.

36. Jacobs BC, van Doorn PA, Schmitz PI, et al: Campylobacter jejuni infections and anti-GM1 antibodies in Guillain-Barré syndrome. Ann Neurol 40: 181-187, 1996.

37. Koga M, Gilbert M, Takahashi M, et al: GQ1b-seronegative Fisher syndrome: clinical features and new serological markers. J Neurol 259: 1366-1374, 2012.

38. Lehmann HC, Lopez PH, Zhang G, Ngyuen T, Zhang J, et al: Passive immunization with anti-ganglioside antibodies directly inhibits axon regeneration in an animal model. J Neurosci 27: 27-34, 2007.

39. Sheikh KA and Zhang G: An update on pathobiologic roles of anti-glycan antibodies in Guillain-Barré syndrome. F1000 Biol Rep 2: 21, 2010. 